04,12

\title{
Влияние ультрадиспергирования на химические сдвиги рентгеновских K-линий в оксидах меди и марганца
}

\author{
() А.А. Набережнов ${ }^{1}$, А.Е. Совестнов ${ }^{2}$, Д.А. Курдюков ${ }^{1}$, Э.В. Фомин ${ }^{2}$, А.В. Фокин ${ }^{1}$ \\ ${ }^{1}$ Физико-технический институт им. А.Ф. Иоффре РАН, \\ Санкт-Петербург, Россия \\ ${ }^{2}$ ПИЯФ им. Б.П. Константинова НИЦ „Курчатовский институт“, \\ Гатчина, Россия \\ E-mail: alex.nabereznov@mail.ioffe.ru
}

Поступила в Редакцию 2 июня 2020 г.

В окончательной редакции 2 июня 2020 г.

Принята к публикации 4 июня 2020 г.

\begin{abstract}
Приведены результаты исследования сдвигов рентгеновских $K_{\alpha}$ - и $K_{\beta}$-линий марганца и меди, полученные для наночастиц оксидов $\mathrm{CuO}, \mathrm{MnO}, \mathrm{Mn}_{3} \mathrm{O}_{4}$ и $\mathrm{MnO}_{2}$ (относительно соответствующих массивных материалов). Наночастицы оксидов синтезированы в порах боросиликатных стекол со средним диаметром пор $7 \pm 1 \mathrm{~nm}$ из соответствующих нитратов, введенных в поры методом капиллярной пропитки. Установлено, что для нанокомпозитов с $\mathrm{CuO}$ и с $\mathrm{Mn}_{3} \mathrm{O}_{4}$ наблюдается небольшое (порядка $\sim 0.1 \mathrm{el} /$ atom) увеличение роли $3 d$-электронов, а для нанокомпозитов с $\mathrm{MnO}_{2}-$ небольшое уменьшение участия этих электронов в химической связи.
\end{abstract}

Ключевые слова:: нанокомпозитные материалы, пористые матрицы, электронная подсистема, спектроскопия рентгеновского излучения, оксиды меди и марганца.

DOI: 10.21883/FTT.2020.10.49912.118

\section{1. Введение}

Исследование свойств и структуры наночастиц в последние годы привлекает пристальное внимание в связи с развитием принципиально новых технологий, использующих ультрадиспергированные материалы и композиты на их основе. К настоящему времени экспериментально установлено, что макроскопические физические свойства и кристаллическая структура целого ряда таких объектов значительно отличаются от свойств аналогичных массивных материалов. Существует много методов получения наночастиц и одним из них является введение (или химический синтез) веществ в поры природных или искусственных пористых матриц. Так, например, для наночастиц галлия $[1,2]$ и индия $[3,4]$ в нанопористых стеклах обнаружены новые, ранее неизвестные, кристаллические фазы. Полученные на основе пористых матриц нанокомпозитные материалы (НКМ) обладают целым рядом макроскопических свойств, привлекательных для практического применения таких, как высокая диэлектрическая проницаемость [5,6], рост ионной проводимости $[7,8]$, расширение диапазона существования сегнетоэлектрической фазы $[9,10]$, резкое увеличение критических магнитных полей для сверхпроводящего состояния $[11,12]$ и т. п. Обнаружено, что в ультрамалых частицах металлов происходит существенная трансформация спектров элементарных возбуждений и изменение констант силовой связи между атомами [13-16]. Естественно не остались без внимания и свойства электронных подсистем ультрадиспергированных веществ: так было показано, что в наночастицах металлов со средним размером 1-2 nm и в структурах типа „ядро-оболочка“ (core-hell) [17-20] происходит размерно-индуцированный переход „металл-диэлектрик“, изменяется плотность состояний вблизи уровня Ферми, увеличивается вклад электронной подсистемы в теплоемкость [21] при низких температурах, происходят сдвиги $3 d-$ и $4 d$-электронных уровней $[20,22,23]$ и т.п. Естественно ожидать, что перестройка электронной подсистемы будет приводить и к изменению (сдвигу) энергии характерных для данного материала линий рентгеновского излучения. Методика измерения малых энергетических смещений рентгеновских линий с помощью кристаллдифракционной спектрометрии [24,25] заключается в том, что это сдвиг уровней измеряется непосредственно и в одинаковых экспериментальных условиях, а не находится как разность двух больших чисел, отвечающих измеренным энергиям рентгеновских линий для двух сравниваемых образцов. Чувствительность метода позволяет напрямую измерять величины сдвигов линий порядка $10^{-3}-10^{-4}$ от их естественной ширины (т. е. величины $\sim 1 \mathrm{meV}$ ). Ранее с помощью этого метода были впервые обнаружены сдвиги рентгеновских $K$-линий у тяжелых элементов $(z=32-74)$ при переходе от одного химического соединения к другому (с разной валентностью), которые до тех пор считались экспериментально неизмеримыми [24]. Более того метод дает возможность однозначно идентифицировать тип электронов, принимающих участие в химический связи, и с высокой точностью определять заселенности внешних (валентных) ор- 
Величины сдвигов линий $K_{\alpha 1}, K_{\alpha 2}, K_{\beta 1,3}$ для массивных и наноструктурированных оксидов $\mathrm{Mn}$ и $\mathrm{Cu}$, а также $4 s-$ и $3 d-$ „факсимиле“ для меди из работы [28].

\begin{tabular}{|c|c|c|c|c|c|}
\hline $\mathrm{N}$ & Образец-репер $S_{\text {ref }}$ & $\operatorname{shift}\left(\Delta\left(K_{\alpha 1}\right)\right), \mathrm{meV}$ & $\operatorname{shift}\left(\Delta\left(K_{\alpha 2}\right)\right), \mathrm{meV}$ & shift $\left(\Delta\left(K_{\beta 1,3}\right)\right), \mathrm{meV}$ & split $\left(\Delta\left(K_{\beta 1,3}-K_{\beta^{\prime}}\right)\right), \mathrm{meV}$ \\
\hline 1 & 2 & 3 & 4 & 5 & 6 \\
\hline 1 & $\mathrm{Cu}, 4 s$-facsimile $[28]$ & $189 \pm 4$ & $255 \pm 3$ & $-148 \pm 4$ & \\
\hline 2 & $\mathrm{Cu}, 3 d$-facsimile [28] & $-110 \pm 5$ & $-168 \pm 5$ & $114 \pm 7$ & \\
\hline 3 & $\mathrm{Mn}($ bulk $)-\mathrm{Mn}_{2} \mathrm{O}_{3}$ (bulk) & $-106 \pm 5$ & $290 \pm 5$ & $763 \pm 8$ & \\
\hline 4 & $\mathrm{MnF}_{2}($ bulk $)-\mathrm{Mn}_{2} \mathrm{O}_{3}($ bulk $)$ & $236 \pm 3$ & $-20 \pm 13$ & $851 \pm 10$ & $15465 \pm 39$ \\
\hline 5 & $\mathrm{MnO}($ bulk $)-\mathrm{Mn}_{2} \mathrm{O}_{3}-($ bulk $)$ & $194 \pm 2$ & $126 \pm 4$ & $318 \pm 5$ & $14409 \pm 46$ \\
\hline 6 & $\mathrm{MnO}($ nano $)-\mathrm{Mn}_{2} \mathrm{O}_{3}($ bulk $)$ & $196 \pm 3$ & $102 \pm 56$ & $419 \pm 11$ & $14537 \pm 111$ \\
\hline 7 & $\mathrm{Mn}_{3} \mathrm{O}_{4}($ bulk $)-\mathrm{Mn}_{2} \mathrm{O}_{3}$ (bulk) & $40 \pm 3$ & $46 \pm 4$ & $47 \pm 6$ & $13970 \pm 82$ \\
\hline 8 & $\mathrm{Mn}_{3} \mathrm{O}_{4}$ (nano) $-\mathrm{Mn}_{2} \mathrm{O}_{3}$ (bulk) & $27 \pm 3$ & $51 \pm 5$ & $-35 \pm 5$ & $13820 \pm 166$ \\
\hline 9 & $\mathrm{MnO}_{2}($ bulk $)-\mathrm{Mn}_{2} \mathrm{O}_{3}($ bulk $)$ & $-215 \pm 5$ & $-78 \pm 5$ & $-445 \pm 5$ & $13075 \pm 143$ \\
\hline 10 & $\mathrm{MnO}_{2}$ (nano) $-\mathrm{Mn}_{2} \mathrm{O}_{3}$ (bulk) & $-195 \pm 6$ & $-70 \pm 6$ & $-394 \pm 4$ & $12594 \pm 364$ \\
\hline 11 & $\mathrm{MnO}($ nano $)-\mathrm{MnO}($ bulk $)$ & $3.4 \pm 1.8$ & $-24 \pm 3$ & $107 \pm 6$ & $14537 \pm 111$ \\
\hline 12 & $\mathrm{Mn}_{3} \mathrm{O}_{4}($ nano $)-\mathrm{Mn}_{3} \mathrm{O}_{4}($ bulk $)$ & $-65 \pm 4$ & $7.7 \pm 6.3$ & $-80 \pm 6$ & $13820 \pm 166$ \\
\hline 13 & $\mathrm{MnO}_{2}($ nano $)-\mathrm{MnO}_{2}($ bulk $)$ & $20 \pm 8$ & $7.3 \pm 7.1$ & $46 \pm 6$ & $12594 \pm 364$ \\
\hline 14 & $\mathrm{Cu}_{2} \mathrm{O}($ bulk $)-\mathrm{Cu}($ bulk $)$ & $131 \pm 5$ & $142 \pm 4$ & $-137 \pm 4$ & $2799 \pm 236$ \\
\hline 15 & $\mathrm{CuO}($ bulk $)-\mathrm{Cu}($ bulk $)$ & $106 \pm 3$ & $107 \pm 2$ & $-95 \pm 4$ & $1724 \pm 197$ \\
\hline 16 & $\mathrm{CuO}($ nano $)-\mathrm{Cu}($ bulk $)$ & $93 \pm 4$ & $98 \pm 5$ & $-94 \pm 5$ & $1354 \pm 184$ \\
\hline 17 & $\mathrm{CuO}($ nano $)-\mathrm{CuO}$ (bulk) & $-15 \pm 3$ & $-19 \pm 6$ & $1.6 \pm 3.8$ & \\
\hline
\end{tabular}

Примечание. В столбцах 3-5 представлены величины сдвигов соответствующих $K$-линий, а в столбце $6-$ величина расщепления $\Delta\left(E\left(K_{\beta 1,3}\right)-E\left(K_{\beta^{\prime}}\right)\right)$ для указанных в столбце 2 пар образец-репер.

биталей атомов, т. е. на микроскопическом уровне исследовать электронные структуры атомов, так как именно изменение заселенности валентных состояний приводит к малому изменению энергий внутренних рентгеновских переходов данного атома [26-28]. Предполагая, что такие малые сдвиги могут присутствовать для наночастиц и/или для наноструктур на основе нанопористых матриц, мы решили применить этот метод и для исследования НКМ. Первые результаты, полученные для наночастиц In, $\mathrm{Pb}, \mathrm{Pd}$ и некоторых оксидов марганца $[4,29,30]$ показали, что наблюдаемые сдвиги, несмотря на их малость, вполне наблюдаемы и статистически хорошо обеспечены. Целью настоящей работы было проведение более детальных исследований влияния ограниченной геометрии (или размерного эффекта) на сдвиги рентгеновских $K$-линий марганца и меди в оксидах этих элементов по сравнению с аналогичными результатами для массивных материалов.

\section{2. Образцы и методика проведения измерений}

В качестве исходных матриц использовались изготовленные в ФТИ им. А.Ф. Иоффе пористые натрий-боросиликатные стекла (PG7) со средним диаметром пор 7(1) nm. Поры в этих стеклах образуют многосвязную дендритную сквозную сеть каналов, общая пористость использованных стекол составляла 22-25\%. Средний диаметр пор определялся с помощью ртутной порометрии. Из пористых стекол готовились тонкие пластины толщиной около $1 \mathrm{~mm}$, которые использовались для дальнейшего заполнения и получения наночастиц.

Введение оксидов марганца $\left(\mathrm{MnO}, \mathrm{MnO}_{2}, \mathrm{Mn}_{3} \mathrm{O}_{4}\right)$ и оксида меди $(\mathrm{CuO})$ в поры осуществлялось в несколько этапов. Пластинки пористого стекла PG7 пропитывались насыщенными водными растворами $\mathrm{Mn}\left(\mathrm{NO}_{3}\right)_{2}$ и $\mathrm{Cu}\left(\mathrm{NO}_{3}\right)_{2}$, соответственно, под действием капиллярных сил. Затем образцы высушивались и отжигались на воздухе при температуре $250^{\circ} \mathrm{C}$ в течение $30 \mathrm{~min}$, при этом нитраты в порах PG7 разлагались до нерастворимых оксидов (а также оксо- и гидроксонитратов), не имеющих определенного химического состава, которые в дальнейшем служили прекурсорами для синтеза целевых веществ. Процедуры капиллярной пропитки и отжига повторялись циклически до достижения требуемой степени заполнения пор.

Для синтеза целевых веществ в порах PG7 проводился отжиг образцов в различных газовых средах. Так, оксиды $\mathrm{CuO}$ и $\mathrm{MnO}_{2}$ синтезировались посредством отжига образцов, заполненных соответствующими прекурсорами, в кислороде, $\mathrm{MnO}$ - в водороде, а $\mathrm{Mn}_{3} \mathrm{O}_{4}-$ в форвакууме (при парциальном давлении кислорода $\sim 10^{-2}$ Torr). Все отжиги проводились при температуре $400^{\circ} \mathrm{C}$ в течение $5 \mathrm{~h}$. Заполнение пор PG7 оксидами составляло порядка $30-40 \%$ от общего порового пространства.

Перед измерением пластины перетирались в порошок, который помещался в специальные капсулы для проведения исследований. Измерения сдвига линий $K_{\alpha 1}, K_{\alpha 2}$ и $K_{\beta 1,3}$ для массивных и наноструктурированных оксидов марганца проводились при комнатной температуре на 
кристалл - дифракционном спектрометре по Иоганну [25] в одних и тех же экспериментальных условиях. В экспериментах определялось относительное смещение линий между массивным и наноструктурированным образцом. Процедура измерений и обработки подробно изложена в работе [25]. Полученные величины сдвигов рентгеновских $K$-линий приведены в таблице. Все сдвиги определялись относительно реперного образца, т.е., например, величина сдвига для пары $\mathrm{Mn}-\mathrm{Mn}_{2} \mathrm{O}_{3}$ $\Delta E\left(K_{\alpha 1}\right)=E_{K \alpha 1}(\mathrm{Mn})-E_{K \alpha 1}\left(\mathrm{Mn}_{2} \mathrm{O}_{3}\right)$, в таблице этот сдвиг обозначен как $\operatorname{shift}\left(\Delta E\left(K_{\alpha 1}\right)\right)$, заголовки колонок в этой же таблице $\operatorname{shift}\left(\Delta E\left(K_{\alpha 2}\right)\right)$ и $\operatorname{shift}\left(\Delta E\left(K_{\beta 1.3}\right)\right.$ соответствуют сдвигам линий $K_{\alpha 2}$ и $K_{\beta 1,3}$. Практически для всех массивных оксидов марганца репером (далее reference sample $-S_{\text {ref }}$ ) служил оксид $\mathrm{Mn}_{2} \mathrm{O}_{3}$. Для наноструктурированных оксидов $\mathrm{Mn}$ соответствующим $S_{\text {ref }}$ был аналогичный массивный оксид. Для оксидов меди в качестве $S_{\text {ref }}$ использовался образец из массивного металла. В таблице представлены результаты, усредненные по нескольким сериям измерений (обычно $\sim 10$ серий).

Все полученные образцы предварительно тестировалась на рентгеновском дифрактометре ДРОН-2 на излучении $K_{\alpha}$ меди.

\section{3. Результаты и обсуждение}

\section{1. Дифракционные спектры для НКМ на основе PG7}

На рис. 1, $a-c$ приведены дифрактограммы для массивных (нижние кривые на каждом рисунке) и синтезированных в порах PG7 (верхние кривые) $\mathrm{MnO}, \mathrm{Mn}_{3} \mathrm{O}_{4}$ и $\mathrm{MnO}_{2}$.

Экспериментальные результаты выделены серым цветом, черными линиями для наглядности прорисованы отражения, соответствующие структурам оксидов марганца и полученные в результате подгонки. Хорошо видно, что структуры $\mathrm{MnO}$ и $\mathrm{Mn}_{3} \mathrm{O}_{4}$ в порах PG7 (рис. $1, a, b$ ) хорошо соответствуют структурам массивных оксидов. Дополнительный пик в области $2 \theta \sim 20^{\circ}$ связан с рассеянием на аморфном $\mathrm{SiO}_{2}$, из которого состоит сама матрица PG7.

Как показали результаты профильного анализа, образец $\mathrm{PG} 7+\mathrm{MnO}_{2}$ (рис. $1, c$ ) является смесью „обычного“ $\alpha-\mathrm{MnO}_{2}$, такого же как в массивном образце, и, даже в большей степени, относительно редко встречающегося $\varepsilon-\mathrm{MnO}_{2}-$ смоделированные спектры для этих двух фаз представлены на рис. 1, $c$ внизу. Аналогичные результаты были получены (рис. 2) для массивного оксида меди (нижняя кривая) и $\mathrm{HKM} \mathrm{PG7}+\mathrm{CuO}$ (верхняя кривая).

Следует отметить, что дифракционные пики для всех НКМ значительно уширены по сравнению с массивными материалами, из анализа этого уширения мы получили оценку размеров наночастиц оксидов в порах, который оказался от 10-12 (для оксидов марганца) до $14.5 \mathrm{~nm}$ для $\mathrm{CuO}$ в порах $\mathrm{PG7}$.
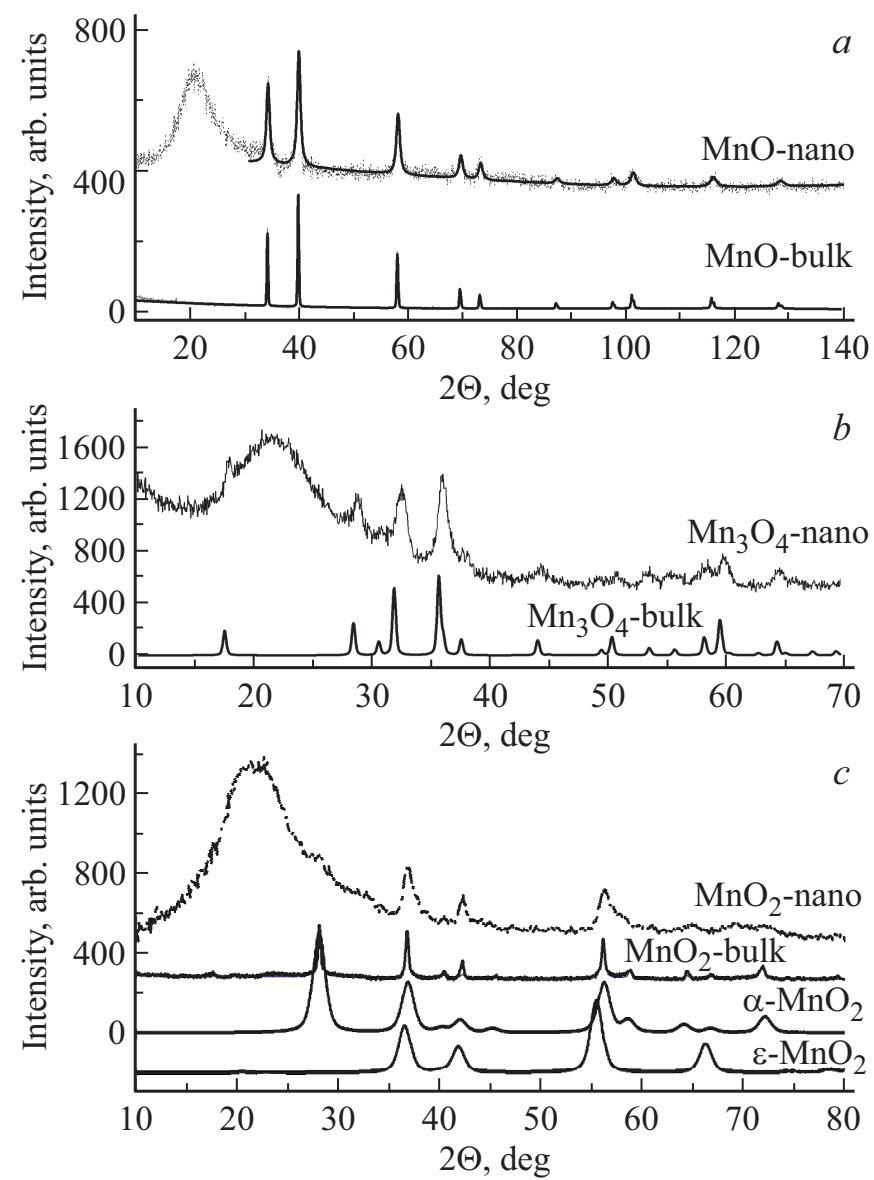

Рис. 1. Экспериментальные дифракционные спектры массивных и наноструктурированных оксидов марганца. $c-$ спектры для $\alpha-\mathrm{MnO}_{2}$ и $\varepsilon-\mathrm{MnO}_{2}$.

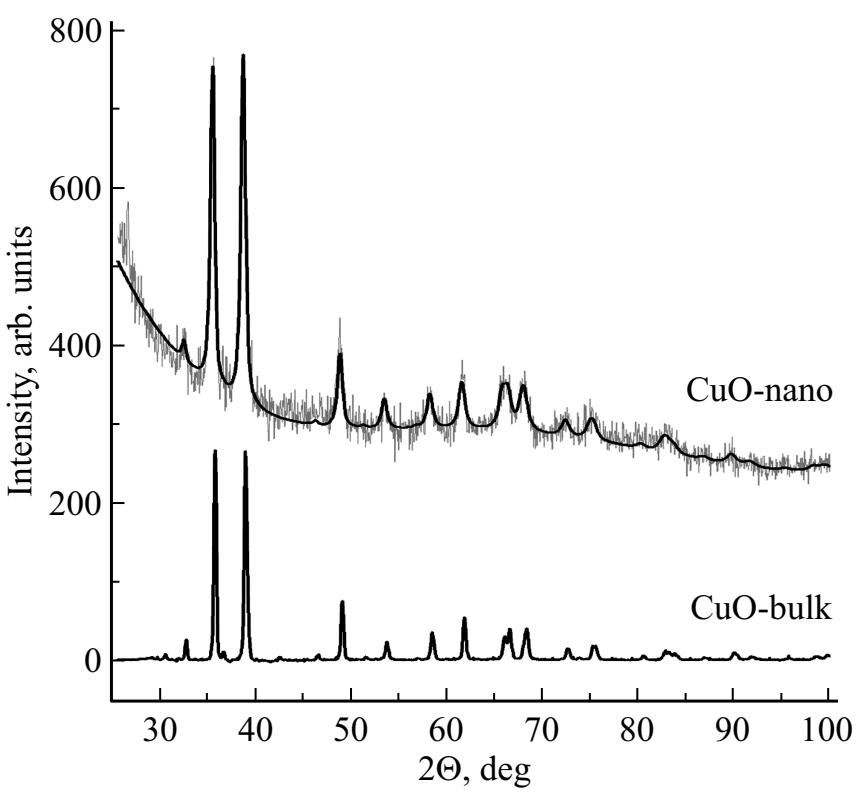

Рис. 2. Экспериментальные дифракционные спектры для массивного и наноструктурированного оксида меди. В случае массивного оксида меди фон вычтен. 
Эти величины хорошо совпадают с размерами, полученными из анализа данных по дифракции нейтронов на этих же образцах [31,32]. Каких-либо посторонних пиков на дифракционных спектрах не наблюдалось, что свидетельствует о том, что в изготовленных НКМ находятся именно интересующие нас соединения.

\section{2. Рентгеновская спектроскопия химических сдвигов $K$-линий оксидов}

Метод смещения рентгеновских линий (дифференциальная эмиссионная рентгеновская спектроскопия), разработанный в работе [33], универсален и обладает высокой избирательностью, что позволяет однозначно и независимо идентифицировать тип электрона $(s, p, d, f)$, принимающего участие в химической связи, а также данный метод с высокой точностью (до $0.01 \mathrm{el} / \mathrm{atom}$ (электрон/атом)) позволяет определить заселенности валентных орбиталей. Еще одним преимуществом этого подхода является то, что полученные результаты относятся ко всему объему образца, а не только к его поверхности. При уходе валентного электрона на образование химической связи происходит малое, но как показано в $[26,33]$ вполне измеримое, смещение по энергии линий $K_{\alpha 1,2}(1 s-2 p), K_{\beta 1,3}(1 s-3 p)$ и $K_{\beta 2,4}(1 s-4 p)$. В этом же обзоре было показано, что электронное состояние элемента в соединении можно характеризовать своеобразным „факсимиле“ - набором смещений диаграммных рентгеновских линий относительно соответствующего металла. В общем случае величина сдвига („факсимиле“) зависит от валентности, степени ионности (ковалентности) элемента в соединении и от спинового состояния $d$-оболочки. Близость „факсимиле“ какого-либо элемента в его двух соединениях свидетельствует о идентичности электронных конфигураций (валентности, ионности и ковалентности) данного (исследуемого) элемента в этих соединениях.

Уместно задать вопрос о том, какие сдвиги рентгеновских линий можно ожидать в случае ультрадиспергированных наночастиц и/или соединений, находящихся в условиях ограниченной геометрии. В первую очередь следует отметить, что для таких образцов (пока будем полагать, что матрица не принимает участия в модификации свойств внедренных материалов, а только формирует условия ограниченной геометрии) характерно большая доля поверхностных атомов (до 30\%) в зависимости от формы наночастицы. Во-вторых, установлено $[13,14,34]$, что локальная симметрия в поверхностных слоях наночастицы (до 3 координационной сферы включительно) может сильно отличаться от симметрии, характерной для массивного материала, что приводит, например, к значительной модификации фононных спектров [13-16], причем при этом явно просматривается зависимость от размера наночастиц $[13,14]$. Естественно, что это может (и будет) приводить к искажению волновых функций внешних электронов атомов в приповерхностных слоях наночастиц. Более того, в случае, когда такие искажения элементарных ячеек относительно велики и простираются на заметную глубину, возможно такое изменение симметрии кристаллической решетки, которое может привести к изменению взаимного положения уровней валентных орбиталей и зон, что, в свою очередь, вызовет перераспределение электронов между зонами в металлах и изменение ковалентности в изоляторах. В этих случаях возможны заметные эффекты сдвигов внутренних уровней и рентгеновских линий. При значительном искажении симметрии кристаллической решетки (т.е. и изменения внутреннего кристаллического поля) возможно изменение и спинового состояния атомов, которое можно наблюдать по изменению расщепления линий $K_{\beta 1,3}$ и $K_{\beta^{\prime}} \Delta\left(E\left(K_{\beta 1,3}\right)-E\left(K_{\beta^{\prime}}\right)\right)$, которое в таблице обозначено как $\operatorname{Split}\left(K_{\beta 1,3}-K_{\beta^{\prime}}\right)$ для приведенных в колонке 2 пар исследованных образцов.

3.2.1. Сдвиги $K$-линий в оксидах меди. Согласно электронной структуре в металлической меди имеется один $4 s$-электрон сверх заполненной $3 d$-оболочки, поэтому величины сдвигов $K$-линий меди (относительно металла) для одновалентного соединения с большой ионностью дает $4 s$-,факсимиле“, а двухвалентного соединения относительно одновалентного - $3 d$-,ффаксимиле“. Эти два параметра были определены ранее в работе [28]. На рис. 3, $a$ представлены измеренные сдвиги $\Delta E\left(K_{\alpha 1}\right), \Delta E\left(K_{\alpha 2}\right)$ и $\Delta E\left(K_{\beta 1,3}\right)$ для массивных $\mathrm{CuO}, \mathrm{Cu}_{2} \mathrm{O}$ и НКМ $\mathrm{CuO}+\mathrm{PG} 7$, на вставке внизу - разности $\Delta E\left(\mathrm{CuO}_{\text {nano }}-\mathrm{CuO}_{\text {bulk }}\right)$ для линий $K_{\alpha 1}, K_{\alpha 2}$ и $K_{\beta 1,3}$. На рис. $3, b-$ „факсимиле“ для $4 s-$ и $3 d$-электронов меди и, для сравнения, никеля [28].

Из рисунка видно, что химическая связь в $\mathrm{Cu}_{2} \mathrm{O}$ осуществляется $4 s$-электронами, причем ионность здесь невелика, т.к. по величине сдвиги для $\mathrm{Cu}_{2} \mathrm{O}$ значительно меньше, чем для $4 s$-,факсимиле“. В процессе дальнейшего окисления (образование $\mathrm{CuO}$ ) добираются оставшиеся $4 s$-электроны и вступают в связь, в соответствии с ионностью, $3 d$-электроны. Из-за противоположного знака „факсимиле“ (сдвигов) $4 s$ - и $3 d$-электронов (рис. $3, b)$, суммарный эффект относительно невелик. Действительно, если бы на образование химической связи в $\mathrm{CuO}$ уходили полностью один $4 s-$ и один $3 d$-электроны, то наблюдаемый сдвиг (согласно „факсимиле“ этих электронов) должен был бы быть, например для $K_{\alpha 1}, \Delta E\left(K_{\alpha 1}\right)=189-110=79(5) \mathrm{meV}$. В эксперименте мы наблюдаем сдвиг $105.5 \pm 3.1 \mathrm{meV}$, т. е. не происходит полного вовлечения $3 d$-электрона в химическую связь.

Как видно из рис. $3, a$, сдвиги $K$-линий меди в $\mathrm{HKM} \mathrm{CuO}+\mathrm{PG} 7$ несколько ниже, чем в массивном $\mathrm{CuO}$, т.е. отрицательный вклад из-за $3 d$-электрона становится больше. При этом разность сдвигов $\Delta E\left(\mathrm{CuO}_{\text {nano }}-\mathrm{CuO}_{\text {bulk }}\right)$ („факсимиле“ чистого эффекта ультрадиспергирования (вставка рис. $3, a$ ) очень похожа на $3 d$-,факсимиле“ по форме. Можно оценить изменение (степень вовлеченности $3 d$-электрона), используя данные для $K_{\alpha 1}$ и $K_{\alpha 2}$, как 

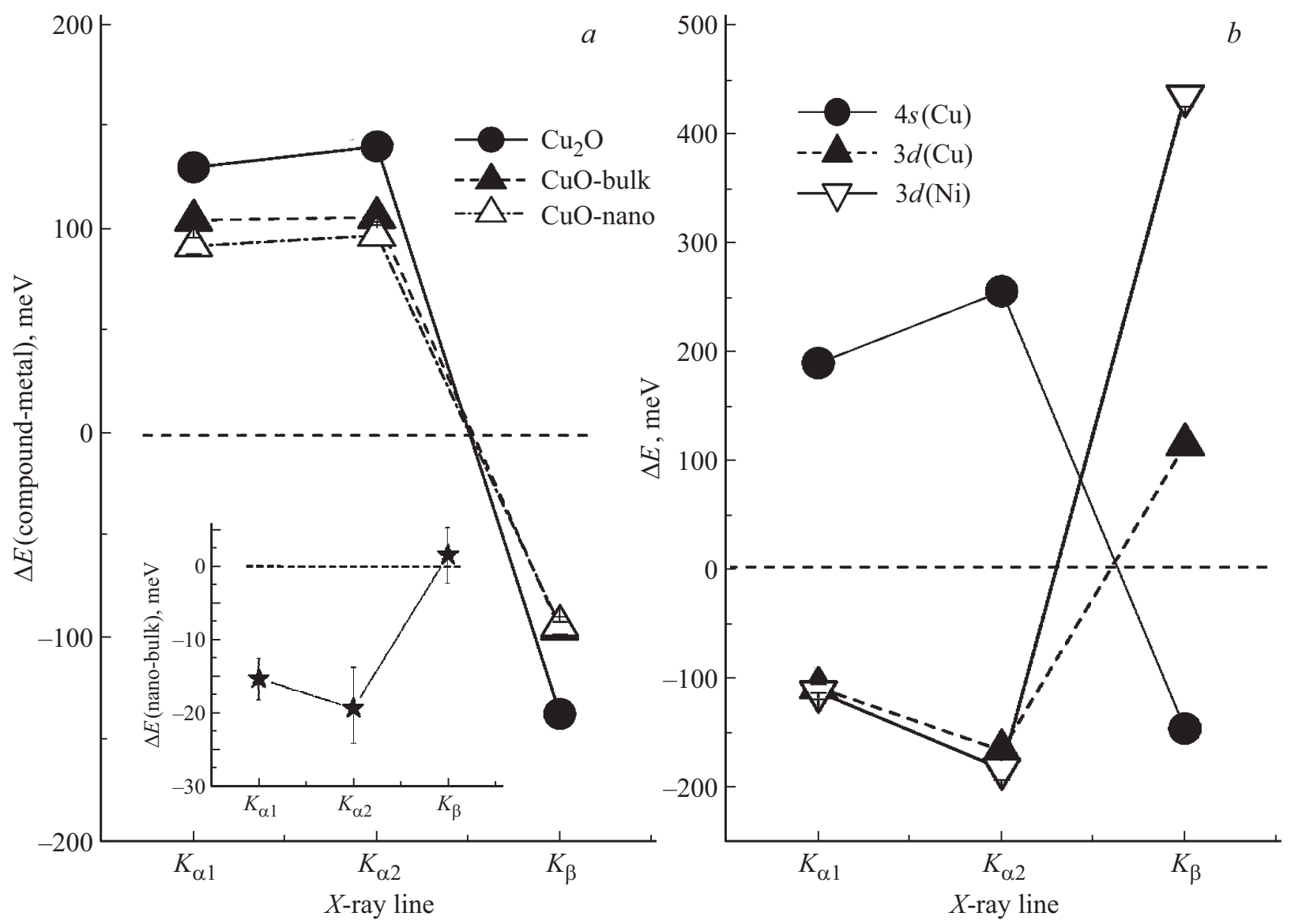

Рис. 3. Экспериментальные сдвиги $K$-линий для оксидов меди $(a)$ и „факсимиле“ $4 s-$ и $3 d$-электронов меди $(b)$ из работы [28].

$\Delta E\left(\mathrm{CuO}_{\text {nano }}-\mathrm{CuO}_{\text {bulk }}\right) / \Delta E(\mathrm{Cu} 3 d$-,,факсимиле“ $)$, что дает величину $\Delta n(3 d)=0.13 \pm 0.03 \mathrm{el} /$ atom. Это означает, что в $\mathrm{HKM} \mathrm{CuO}+\mathrm{PG} 7$ в химической связи принимает участие на $0.13 \pm 0.033 d$-el/atom больше, чем в массивном CuO. Следует заметить, что используемые здесь и далее „факсимиле“ получены экспериментально и при их вычислении не учтены ионности и/или ковалентности используемых соединений, а эти факторы могут изменить величины приведенных сдвигов „факсимиле“, т. е. и оценки $\Delta n(3 d)$, по-видимому, вплоть до 20-30\%. Подчеркнем, что бо́льшие по величине сдвиги $K$-линий меди для НКМ по сравнению с массивными ни в коей мере не означают и бо́льшую валентность. Валентность и степень окисления $\mathrm{Cu}$ в $\mathrm{CuO}$ была и остается равной двум, просто меняется ионность (иначе, степень ковалентности и гибридизация) данной химической связи.

3.2.2. Сдвиг $K$-линий для оксидов марганца. На рис. 4, $a-c$ представлены экспериментальные сдвиги рентгеновских $K$-линий марганца от типа линий, валентности $\mathrm{Mn} \mathrm{и} \mathrm{расщепления} K_{\beta 1,3}-K_{\beta^{\prime}}$-линий для окислов $\mathrm{MnO}, \mathrm{Mn}_{3} \mathrm{O}_{4}$ и $\mathrm{MnO}_{2}$.

Из рис. 4, $a$ видно, что наблюдаемые сдвиги для окислов марганца существенно иные по форме, чем оксидов меди (рис. 3, $a$ ), но одинаковым образом зависят от валентности - увеличение валентности приводит к отрицательным сдвигам всех линий. При этом от величины валентности марганца в этих соединениях и сдвиги $K$-линий и расщепление $K_{\beta 1,3}-K_{\beta^{\prime}}$-линий зависят практически линейно, что обусловлено связью этих параметров с заселенностью $3 d$-орбиталей. Отметим, что большие величины расщепления $K_{\beta 1,3}-K_{\beta^{\prime}}$-линий свидетельствуют о высоко-спиновом состоянии атомов (ионов) марганца в этих окислах, которое подтверждается литературными данными по магнитным моментам марганца, расщеплениями $K_{\beta 1,3}-K_{\beta^{\prime}}$-линий и $3 s$-уровня аналогичных атомов [35-39].

Как можно видеть из рис. 4, $c$, ультрадиспергирование окислов марганца не приводит к изменению спинового (т.е. и магнитного) состояния атомов $\mathrm{Mn}$, поскольку величины расщепления $K_{\beta 1,3}-K_{\beta^{\prime}}$-линий НКМ и массивных аналогичных образцов находятся фактически в пределах одной ошибки измерения. Заметим, что в принципе эффекты изменения спинового состояния очень велики, так, например, изменение высоко-спинового состояния двухвалентного марганца в $\mathrm{MnO}$ (суммарный спин $S=5 / 2)$ до средне-спинового $(S=3 / 2)$ привело бы к уменьшению $\operatorname{Split}\left(K_{\beta 1,3}-K_{\beta^{\prime}}\right)$ от $\sim 14.5$ до $\sim 13 \mathrm{eV}$ (рис. $4, c)$. Разумеется, что не заметить такие изменения было бы невозможно. Комментарий по поводу спинового состояния приведен в части 3.3.3 настоящей статьи. 

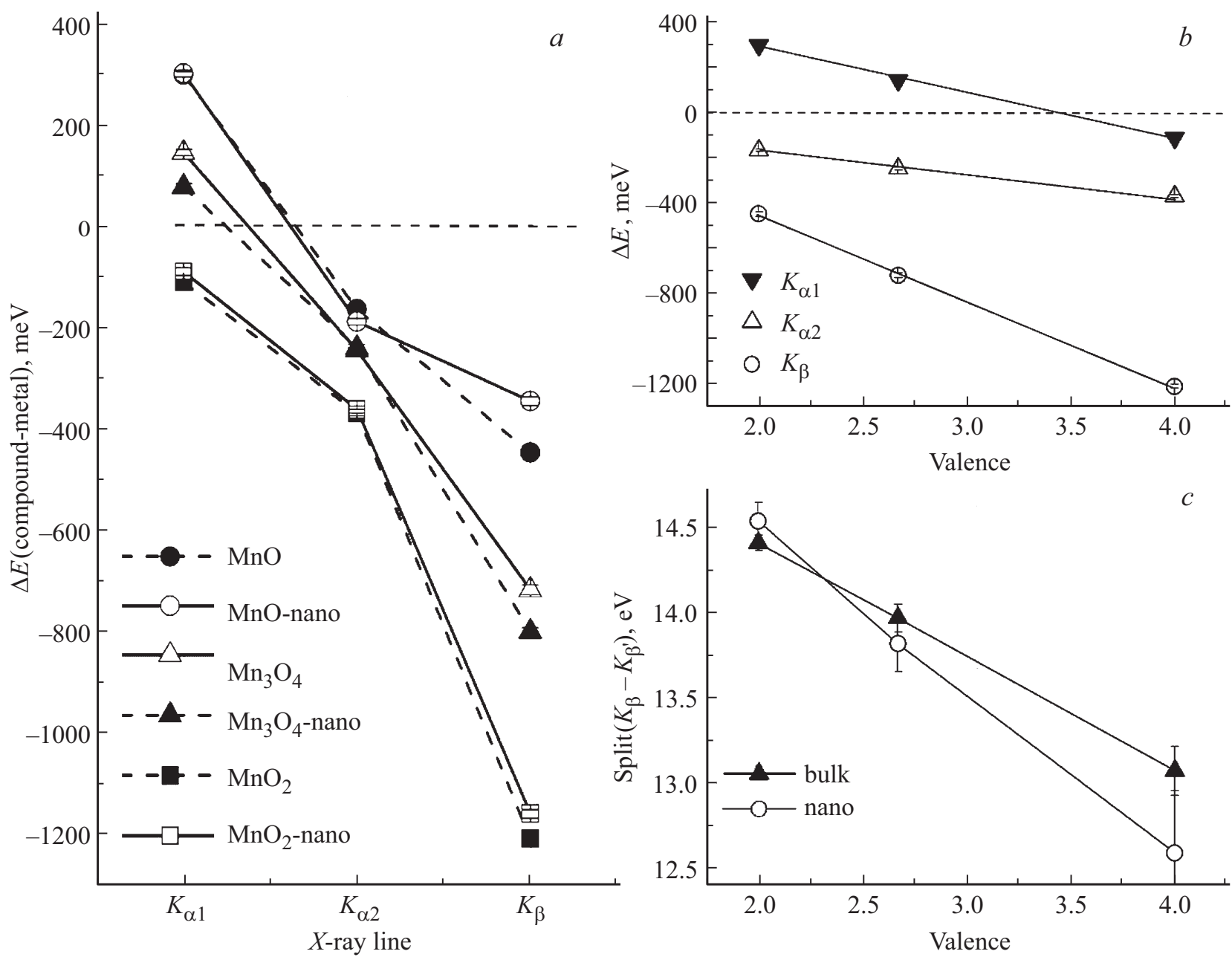

Рис. 4. Экспериментальные зависимости сдвигов рентгеновских $K$-линий марганца от типа линий $(a)$ и валентности $(b)$, а также расщепления $K_{\beta}-K_{\beta^{\prime}}$-линий $(c)$ для массивных и наноструктурированных окислов $\mathrm{MnO}, \mathrm{Mn}_{3} \mathrm{O}_{4}$ и $\mathrm{MnO}_{2}$.

На рис. 4, $а$ явно видно проявление ультрадиспергирования - сдвиги для НКМ и массивных (bulk) образцов заметно отличаются практически во всех точках. Для получения „чистого“ эффекта мы провели прямые измерения сдвигов $\Delta E$ (nano-bulk), результаты которых представлены на рис. 5, $a$. Как можно видеть из рис. 5, $a$, при переходе от массивных к наноструктурированным образцам для оксидов марганца наблюдаются статистически достоверные изменения смещений рентгеновских $K$-линий Mn, в общем случае разной направленности. Для выяснения физико-химического смысла таких изменений их следовало бы сравнить с факсимиле $4 s$ и $3 d$-электронов марганца, но таковых пока нет, а имеющиеся для $\mathrm{Cu}$ и $\mathrm{Ni}$ (рис. $3, b$ ), вряд ли будут подходящими из-за дальности расстояния от $\mathrm{Mn}$ до $\mathrm{Cu}$ и фактически противоположности возможных спиновых (магнитных) состояний.

Однако, как было указано выше, все изменения валентного состояния в рассматриваемых окислах обусловлены заселенностью только $3 d$-орбиталей. Поэтому можно полагать, что и при ультрадиспергировании из- менения химической связи будут обусловлены изменением степени участия в ней именно $3 d$-электронов. Таким образом, нам будет достаточно только „факсимиле“ $3 d$-электронов марганца, которое, очевидно, можно получить из разности сдвигов $K$-линий марганца двух окислов разной валентности. Такие разности $\Delta E\left(\mathrm{Mn}_{3} \mathrm{O}_{4}-\mathrm{MnO}\right)$ и $\Delta E\left(\mathrm{MnO}_{2}-\mathrm{Mn}_{3} \mathrm{O}_{4}\right)$ представлены на рис. $5, b$, из которого можно видеть, что они подобны по форме с хорошей точностью, причем отношение сдвигов $\Delta E\left(\mathrm{MnO}_{2}-\mathrm{Mn}_{3} \mathrm{O}_{4}\right)$ к $\Delta E\left(\mathrm{Mn}_{3} \mathrm{O}_{4}-\mathrm{MnO}\right)$ равно $r(\Delta E)=1.71 \pm 0.07$. Поскольку отношение разностей валентностей $r(\Delta v)=\Delta v\left(\mathrm{MnO}_{2}-\mathrm{Mn}_{3} \mathrm{O}_{4}\right) / \Delta v\left(\mathrm{Mn}_{3} \mathrm{O}_{4}-\right.$ $\mathrm{MnO})=1.33 / 0.67=2$, то, по-видимому, можно утверждать, что отличие $r(\Delta E)$ от двух обусловлено уменьшением ионности атомов марганца по мере увеличения его валентности в окислах. Это дает основание принять, в первом приближении, кривые на рис. 5, $b$ за „факсимиле“ $3 d$-электронов марганца по форме, но заниженное по величине. Из сравнения кривых рис. 5, $a, b$, по-видимому, можно сделать вывод, что „факсимиле“ для $\mathrm{HKM} \mathrm{c} \mathrm{Mn}_{3} \mathrm{O}_{4}$ и „факсимиле“ $3 d$-электронов 
марганца имеют близкие ( $\Lambda$-образные) формы, откуда можно заключить, что в НКМ $\mathrm{Mn}_{3} \mathrm{O}_{4}+$ PG7 по сравнению с массивным в кристаллохимической связи участвует большая доля $3 d$-электронов. Из отношений сдвигов этих кривых оценка этой доли составляет $\Delta_{3 d}\left(\mathrm{Mn}_{3} \mathrm{O}_{4}\right)=0.21 \pm 0.06 \mathrm{el} /$ atom. Напомним, что такие факсимиле $3 d$-электронов марганца имеют заниженное значение, т.е. полученная величина $\Delta_{3 d}\left(\mathrm{Mn}_{3} \mathrm{O}_{4}\right)$ скорее всего завышена. То же самое относится и к другим случаям. В случаях $\mathrm{MnO}$ и $\mathrm{MnO}_{2}$ факсимиле разности $\mathrm{HKM}$ и массивных образцов обратны по форме к факсимиле $3 d$-электронов ( $V$-образны), что соответствует уменьшению участия $3 d$-электронов марганца при ультрадиспергировании этих окислов. Оценки величин этих изменений составляют $\Delta_{3 d}(\mathrm{MnO})=-0.05 \pm 0.09 \mathrm{el} / \mathrm{atom}$ и $\Delta_{3 d}\left(\mathrm{MnO}_{2}\right)=-0.11 \pm 0.01 \mathrm{el} /$ atom, причем большая ошибка оценки в первом случае в существенной мере связана с заметной разницей форм кривых для $\mathrm{MnO}$ на рис. $5, a$ и „факсимиле“ $3 d$-электронов на рис. $5, b$.

3.2.3. Спиновое состояние. В общем случае $3 d$ атомы в соединениях имеют магнитный момент. По его величине магнитные состояния атома делятся на высокоспиновые (электронная конфигурация $3 d(\uparrow)^{5}$ и магнитный момент $\mu \approx 5 \mu \mathrm{B}$ для $\mathrm{MnO}$, например), сред-
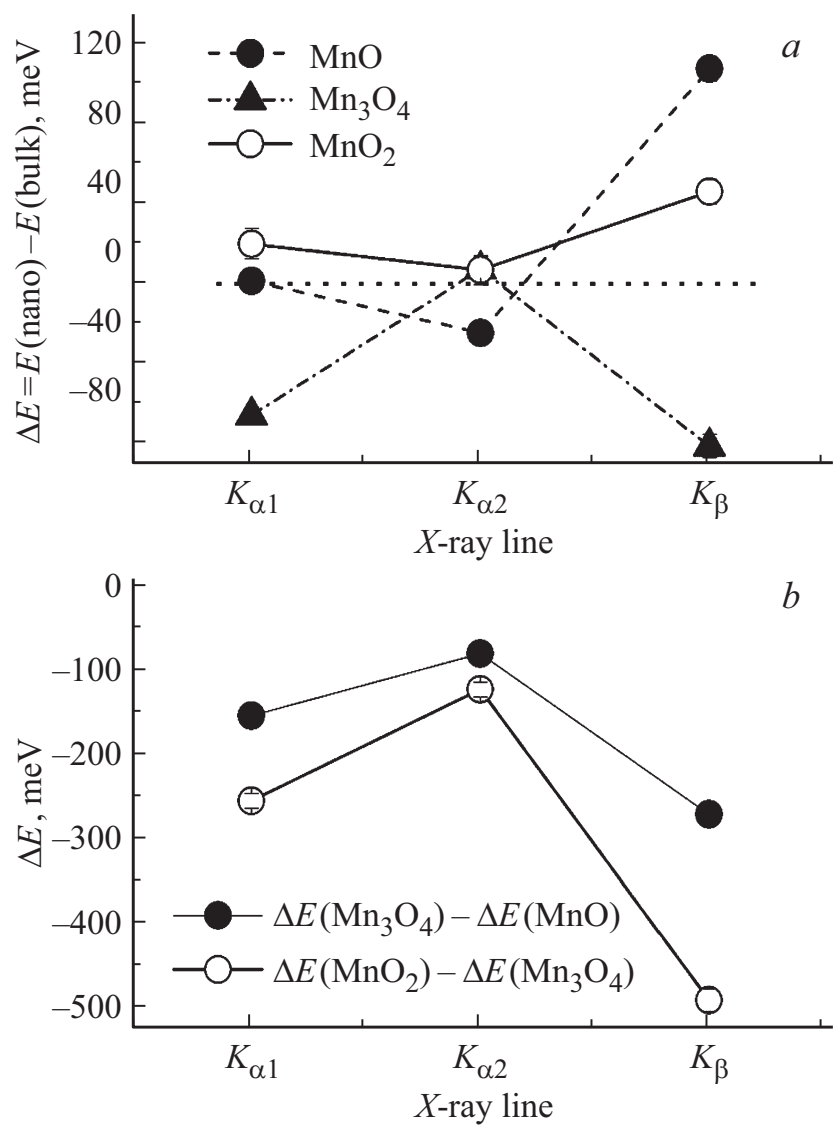

Рис. 5. Изменение сдвигов $K$-линий марганца при ультрадиспергировании его окислов $(a)$ и при изменении валентности марганца $(b)$.

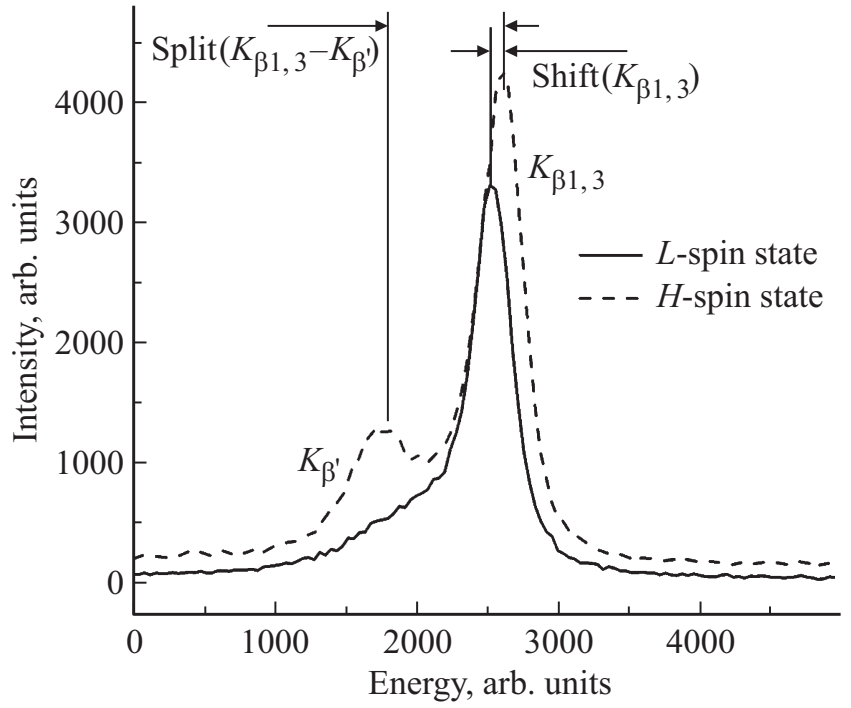

Рис. 6. Профиль $K_{\beta}$-линии $3 d$-элемента с указанием параметpoв $\operatorname{Split}\left(K_{\beta 1,3}-K_{\beta^{\prime}}\right)$ и $\operatorname{Shift}\left(K_{\beta 1,3}\right)$, приведенных в тексте и в таблице. $L$-spin - низко-спиновое состояние, $H$-spin — высокоспиновое состояние.

неспиновые $\left(3 d(\uparrow)^{4} 3 d(\downarrow)^{1}, \mu \approx 3 \mu_{\mathrm{B}}\right)$ и низко-спиновые $\left(3 d(\uparrow)^{3} 3 d(\downarrow)^{2}, \mu \approx 1 \mu_{\mathrm{B}}\right)$. Высоко- и низко-спиновые состояния $3 d$-атомов даже визуально различимы по форме спектра в области $\left(K_{\beta 1,3}-K_{\beta^{\prime}}\right)$-линий (рис. 6$)$, количественную оценку их $\left(\operatorname{Split}\left(K_{\beta 1,3}-K_{\beta^{\prime}}\right)\right)$ можно получить и из подходящей аппроксимации этого спектра. Чаще всего считается, что это расщепление обусловлено обменным взаимодействием между $3 p$ - и $3 d$-оболочками. Поэтому величина расщепления связана сугубо с полным спином, т.е. с магнитным моментом, атома. Макроскопическое (кооперативное) состояние вещества (ферромагнитное, антиферромагнитное, парамагнитное и т.д.) на $\operatorname{Split}\left(K_{\beta 1,3}-K_{\beta^{\prime}}\right)$ практически не влияют.

\section{4. Заключение}

Показано, что кристаллическая структура оксидов $\mathrm{MnO}, \mathrm{Mn}_{3} \mathrm{O}_{4}$ и $\mathrm{CuO}$ в массивном состоянии и в НКМ на основе PG7 практически одинаковы, в случае НКМ PG7- $\mathrm{MnO}_{2}$ наблюдается смесь фаз: обычно свойственной массивному материалу $\alpha-\mathrm{MnO}_{2}$ и довольно экзотической $\varepsilon-\mathrm{MnO}_{2}$. Из анализа смещений рентгеновских $K$-линий установлено, что для $\mathrm{HKM} \mathrm{с} \mathrm{CuO}$ и с $\mathrm{Mn}_{3} \mathrm{O}_{4}$ наблюдается небольшое (порядка $\sim 0.1 \mathrm{el} /$ atom) увеличение роли $3 d$-электронов в химической связи, а в $\mathrm{HKM} \mathrm{PG7}+\mathrm{MnO}_{2}$ - небольшое уменьшение участия $3 d$-электронов в химической связи. Ситуация с НКМ, содержащих наночастицы $\mathrm{MnO}$, пока неоднозначна и требует дальнейшего изучения и анализа, так как нельзя исключить значительного изменения ковалентности по сравнению с массивным образцом. Общая тенденция величин сдвигов $K$-линий для массивных оксидов соответствуют ожидаемым при изменении валентности 
марганца от 0 до +4 , если исходить из подхода, изложенного в работе [24]. При этом величины сдвигов всех $K$-линий линейно зависят от валентности марганца, а отрицательная величина наклона этих прямых однозначно свидетельствует, что увеличение степени окисления Мn обусловлено уменьшением заселенности $3 d$-орбитали. Разнонаправленное смещение $K$-линий марганца при переходе от металла к моноокислу хорошо объясняется тем, что при этом химическая связь осуществляется смесью $3 d$ - и $4 s$-состояний. Из таблицы также хорошо видно, что присутствует значительная хорошо статистически обеспеченная разница сдвигов энергий $K$-линий между наноструктурированными и массивными оксидами марганца с одинаковой валентностью. Следует подчеркнуть, что эти эффекты не имеют регулярной зависимости от того, какой электрон уходит на образование связи. По-видимому, происходящая перестройка электронной системы связана не только с ультрадиспергированием (с размером наночастиц), но и с особенностями взаимодействия наночастиц оксидов марганца (возможно и меди) с самой матрицей. Здесь необходимо отметить, что использованный метод дает результаты, усредненные по всему объему наночастиц. В то же время естественно полагать, что электронные свойства внутренних областей наночастиц не отличаются от свойств массивных материалов, а все отличия наблюдаются в интерфейсном слое. Толщину этого поверхностного слоя с измененным по сравнению с массивным материалом окружением можно положить равным радиусу третьей координационной сферы согласно работе [13,14,34], в таком случае в наших НКМ (учитывая их дифракционный размер) в этих слоях может находиться до 30\% атомов. Поэтому полученные величины следует считать оценками снизу.

\section{Финансирование работы}

А.В. Фокин благодарит РФФИ (грант 19-02-00760), a А.А. Набережнов - грант РФФИ БРИКС-т 19-5280019 за частичную финансовую поддержку. Разработка методов синтеза оксидных наночастиц выполнена с использованием средств госбюджета по госзаданию 00402019-0012.

\section{Конфликт интересов}

Авторы заявляют, что конфликт интересов у них отсутствует

\section{Список литературы}

[1] И.Г. Сорина, С. Tien, Е.М. Чарная, Ю.А. Кумзеров, Л.А. Смирнов. ФТТ 40, 1552 (1998).

[2] E.V. Charnaya, C. Tien, K.J. Lin, Yu.A. Kumzerov. Phys. Rev. B 58, 11809 (1998).

[3] B. Balamurugan, F.E. Kruis, S.M. Shivaprasad, O. Dmitrieva, H. Záhres. Appl. Phys. Lett. 86, 083102 (2005).
[4] А.А. Набережнов, А.Е. Совестнов, А.В. Фокин. ЖТФ 81, 49 (2011).

[5] S.V. Pan'kova, V.V. Poborchii, V.G. Solov'ev. J. Phys.: Condens. Matter. 8, L203 (1996).

[6] E.V. Colla, E.Yu. Koroleva, Yu.A. Kumzerov, B.N. Savenko, S.B. Vakhrushev. Ferroelectr. Lett. 20, 143 (1996).

[7] J. Maier. Nature Mater. 4, 805 (2005).

[8] N. Sata, K. Eberman, K. Eberl. J. Maier. Nature 408, 946 (2000).

[9] S.V. Baryshnikov, E.V. Charnaya, A.Yu. Milinskiy, Yu.A. Shatskaya, C. Tien, D. Michel. Physica B 405, 3299 (2010).

[10] A. Naberezhnov, E. Koroleva, A. Sysoeva, S. Vakhrushev, E. Rysiakiewicz-Pasek, M. Tovar. Proceedings of 6th Int. Conf. on Broadband Dielectric Spectroscopy and applications. Madrid (2010). C. 126.

[11] Г.Х. Панова, А.А. Набережнов, А.В. Фокин. ФТТ 50, 1317 (2008).

[12] А.А. Шиков, М.Г. Землянов, П.П. Паршин, А.А. Набережнов, Ю.А. Кумзеров. ФТТ 12, 2218 (2012).

[13] P.M. Derlet, R. Meyer, L.J. Lewis, U. Stuhr, H. van Swygenhoven. Phys. Rev. Lett. 87, 205501 (2001).

[14] P.M. Derlet, H. van Swygenhoven. Phys. Rev. Lett. 92, 035505 (2004).

[15] П.П. Паршин, М.Г. Землянов, Г.Х. Панова, А.А. Шиков, А.А. Набережнов, Ю.А. Кумзеров, И.В. Голосовский, А.С. Иванов. ЖЭТФ 138, 1127 (2010).

[16] П.П. Паршин, М.Г. Землянов, Г.Х. Панова, А.А. Шиков, Ю.А. Кумзеров, А.А. Набережнов, И. Сергеев, W. Crichton, А.И. Чумаков, R. Ruffer. ЖЭТФ 141, 502 (2012).

[17] M. Rosenblit, J. Jortner. J. Phys. Chem. 98, 9365 (1994).

[18] R. Busani, M. Folkers, O. Chesnovsky. Phys. Rev. Lett. 81, 3836 (1998).

[19] C.P. Vinod, G.U. Kulkarni, C.N.R. Rao. Chem. Phys. Lett. 289, 329 (1998).

[20] C.N.R. Rao, G.U. Kulkarni, A. Govindaraj, B.C. Satishkumar, P.G. Thomas. Pure Appl. Chem. 72, 21 (2000).

[21] А.А. Шиков, Г.Х. Панова, М.Г. Землянов, П.П. Паршин, Ю.А. Кумзеров, А.А. Набережнов, Д.С. Шайтура. ФТТ 53, 2389 (2011).

[22] J.N. Andersen, D. Hennig, E. Lundgren, M. Methfessel, R. Nyholm, M. Scheffle. Phys. Rev. B 50, 17525 (1994).

[23] Chang Q. Sun. Phys. Rev. B 69, 045105 (2004).

[24] О,И. Сумбаев. ЖЭТФ 49, 1716 (1969).

[25] А.А. Петрунин, А.Е. Совестнов, А.В. Тюнис, Э.В. Фомин. Письма в ЖТФ 35, 42 (2009).

[26] О,И. Сумбаев. УФН 124, 281 (1978).

[27] А.Е. Совестнов, А.В. Тюнис, Э.В. Фомин, А.А. Петрунин, А.И. Курбаков, Б.Т. Мелех. Письма в ЖТФ 54, 55 (2012).

[28] А.В. Иванюта, А.Е. Совестнов, Э.В. Фомин. ФТТ 54, 647 (2012).

[29] А.Е. Совестнов, А.А. Набережнов, Ю.А. Кумзеров, А.А. Сысоева, В.А. Ганжа, А.И. Егоров, Н.М. Окунева, В.И. Федоров, Э.В. Фомин. ФТТ 55, 766 (2013).

[30] А.А. Набережнов, А.А. Петрунин, А.Е. Совестнов, Д.А. Курдюков, Э.В. Фомин, С.Б. Вахрушев. Письма в ЖТФ 41, 89 (2015).

[31] I.V. Golosovsky, I. Mirebeau, G. André, D.A. Kurdyukov, Yu.A. Kumzerov, S.B. Vakhrushev. Phys. Rev. Lett. 86, 5783 (2001). 
[32] И.В. Голосовский, А.А. Набережнов, Д.А. Курдюков, І. Мirebeau, G. André. Кристаллография 56, 170 (2011).

[33] O.I. Sumbaev. In collect. Art.: Modern physics in chemistry / Ed. E. Fluck, V.L. Goldanskii. Academic Press. London-N.Y.San Francisco (1976). 406 p.

[34] A. Kara, T.S. Rahman. Phys. Rev. Lett. 81, 1453 (1998).

[35] S. Hirai, A.M. dos Santos, M.C. Shapiro, J.J. Molaison, N. Pradhan, M. Guthrie, C.A. Tulk, I.R. Fisher, W.L. Mao. Phys. Rev. B 87, 014417 (2013).

[36] J. Strempfer, U. Rütt, S.P. Bayrakci, Th. Brückel, W. Jauch. Phys. Rev. B 69, 014417 (2004).

[37] S. Limandri, S. Ceppi, G. Tirao, G. Stutz, C.G. Sanchez, J.A. Riveros. Chem. Phys. 367, 93 (2010).

[38] S.D. Gamblina, D.S. Urchb. J. Electron Spectroscopy Related Phenomena 113, 179 (2001).

[39] В.Р. Галахов, М.К. Фалуб, К. Кюппер, М. Нойманн. Журн. структурной химии 49, S58 (2008).

Редактор Д.В. Жуманов 